\title{
Characterizing Scalability Issues in Spreadsheet Software using Online Forums
}

Kelly Mack

University of Illinois at

Urbana-Champaign

Champaign, IL 61820, USA

knmack2@illinois.edu

John Lee

University of Illinois at

Urbana-Champaign

Champaign, IL 61820, USA

lee98@illinois.edu

\section{Kevin Chang}

University of Illinois at

Urbana-Champaign

Champaign, IL 61820, USA

kcchang@illinois.edu

Permission to make digital or hard copies of all or part of this work for personal or classroom use is granted without fee provided that copies are not made or distributed for profit or commercial advantage and that copies bear this notice and the full citation on the first page. Copyrights for components of this work owned by others than ACM must be honod. Abstracts to post on servers or to redistribute to lists, requires prior specific permission and/or fee. Request permissions from Permissions@acm.org.

C 20 EXM. ISBN $978-1,4503-5621-318184 \$ 15.00$
Karrie Karahalios

(Illinois at

a-Champaign

Aditya Parameswaran

University of Illinois at

Urbana-Champaign

Champaign, IL 61820, USA

adityagp@illinois.edu
Champaign, IL 61820, USA

https://doi.org/10.1145/3170427.3174359

\begin{abstract}
In traditional usability studies, researchers talk to users of tools to understand their needs and challenges. Insights gained via such interviews offer context, detail, and background. Due to costs in time and money, we are beginning to see a new form of tool interrogation that prioritizes scale, cost, and breadth by utilizing existing data from online forums. In this case study, we set out to apply this method of using online forum data to a specific issue - challenges that users face with Excel spreadsheets. Spreadsheets are a versatile and powerful processing tool if used properly. However, with versatility and power come errors, from both users and the software, which make using spreadsheets less effective. By scraping posts from the website Reddit, we collected a dataset of questions and complaints about Excel. Specifically, we explored and characterized the issues users were facing with spreadsheet software in general, and in particular, as resulting from a large amount of data in their spreadsheets. We discuss the implications of our findings on the design of next-generation spreadsheet software.
\end{abstract}

\section{Author Keywords}

Reddit; Scalability; Spreadsheets; Excel 


\section{Introduction}

Spreadsheets are widely used by professionals in a variety of disciplines. Even in their most basic form, they act as a useful organizational aid, allowing users to record information in a two-dimensional tabular layout. With capabilities like formulae and macros to support complex calculations or automate processes, spreadsheets become indispensable as a comprehensive medium for data management and analysis [25].

At the same time, spreadsheets have problems: studies have shown that they foster errors [22, 24], they are not able to easily express useful data operations, such as joins or filters $[5,6]$, and they are known to be sluggish or crash when operating on very large datasets [25]. The last class of problems are what we call scalability problems, i.e., situations where users are unable to complete standard operations in a reasonable amount of time when the scale or complexity of the dataset increases [2]. With the increasing availability of large volumes of data in most domains, we suspect that scalability problems are only going to become more common-as an example, our biologist collaborators routinely use spreadsheets, and can now generate large genomic (VCF) files, but are unable to open these files to verify correctness in spreadsheet software [7]

We conduct a case study of problems in spreadsheet software, with a special emphasis on scalability problems. Prior studies on spreadsheet software have largely focused on the ability of users to use a spreadsheet effectively, as opposed to spreadsheet software problems, including understanding users' conceptual models $[18,20,22]$, and detecting and categorizing user errors $[4,8,15,23]$. In contrast, we focus on settings where spreadsheet software does not meet expectations. For example, one user performed a copy/paste operation on a few hundred thousand rows of data; they waited two and a half hours and still could not accomplish their task. This behavior-where expectations and models of use are clear-is relatively unexplored.

A second difference with prior studies on understanding the use of spreadsheet software is our methodology. Our first attempt at characterizing spreadsheet problems was via interviews with current or aspiring spreadsheet users, such as our biologist collaborators, teaching faculty who managed MOOC student grades in spreadsheets, business analysts wanting to use spreadsheets to study ad campaigns, among others. While these interviews were illuminating in their depth of detail, they were lacking breadth-we wanted to survey a broad population of spreadsheet users, specifically Microsoft Excel users, to understand their problems. Thus, we turned to online forums, specifically Reddit, that captured users' self-reported opinions, questions, and frustrations on the Microsoft Excel spreadsheet subreddit ${ }^{1}$.

To the best of our knowledge, online forums have not been used to understand the spectrum of problems in spreadsheet software. Online forums have been used for other goals, such as understanding the factors that (1) influence perception-e.g., the discovery of credibility factors in medical crowdfunding sites using Reddit [13], (2) describe use and behavior practices-e.g., physical interaction of users with disabilities using YouTube videos [3], and (3) highlight areas for improvement via "app mining"-prioritizing application bug fixes using Google Play Store reviews [12]. While our work is similar in its categorization of improvement areas to [12], they focus on improving an existing code/review pipeline to determine where to modify code, whereas we investigate the scalability landscape surrounding multiple existing use cases in spreadsheets.

${ }^{1}$ http://reddit.com/r/excel 
To accomplish this, we collected over 700 posts from an Excel Reddit forum. While analyzing the posts, four main types of operations emerged that posed problems, both in scalability, and otherwise-importing, managing, querying, and presenting data. We dig deeper to characterize these problem areas to highlight concrete areas of improvement for spreadsheet software, with an eye towards expanding the reach and usability of spreadsheets, especially for very large and complex datasets.

The contributions of this paper are (1) a mapping of challenges users face using spreadsheets in general, as well as (2) how they pertain to scalability, and (3) a methodology for broad evaluation of problems in features, capabilities, and intent specification for end-user software, and (4) a discussion of how to fix these problems, as a means towards building a more robust, scalable, powerful spreadsheet tool.

\section{Related Work}

Our work builds on prior work in two different research areas, (1) spreadsheets and (2) the use of online community discourse as a primary data source for study.

Prior work in spreadsheets

Previous research in spreadsheets have focused on improving spreadsheets through understanding existing problems via user studies and analyzing existing spreadsheets. Researchers have conducted user studies to understand users' conceptual models of spreadsheets to identify how the cognitive process can affect error rates [22], to see how users navigate large spreadsheets [18], to evaluate how multiple users interact with a single spreadsheet [20], and to characterize the strengths and weaknesses of spreadsheets $[9,21]$. Other studies have focused on errors: Powel et al. explore different types of errors that occur and how they can be minimized [23], while others study real spread- sheets to discover errors $[4,15]$. Our approach is instead to identify scalability problems in spreadsheets by exploring troubleshooting posts on an online forum.

Using online communities as a data source

The availability of diverse and large aounts of online community data has led researchers to mine this data to answer research questions $[3,10,11,13,16]$. While this method may bias the user sample to users with internet access and a level of technology savviness, prior works have successfully created rich characterizations of users via this approach. In addition to the papers mentioned in the introduction [3, 12,13], Kulshrestha et al. [16] measured the political bias of an individual Twitter search result by extracting features from the Twitter user's account, while Keelan et al. [11] extracted Youtube videos to measure the sentiment (positive/negative) surrounding immunization.

\section{Methodology}

One goal of this work is to understand the breadth of issues users experience while using Excel, specifically issues related to scalability. To accomplish this, we chose to analyze posts from the Excel forum "/r/excel" on Reddit. There are other spreadsheet related forums such as $/ \mathrm{r} / \mathrm{spreadsheets}$ and $/ r /$ sheets. However, as Microsoft Excel is one of the largest players in the spreadsheet industry, we limited our scope to the Excel forum. Similarly, there are other forums we could have used including StackOverflow or Excel Forum. We chose Reddit due to its popularity and its extensive API.

Constructing and coding a Dataset from Reddit

We collected two datasets of posts from the /r/excel forum using the Reddit API: one dataset to understand the general scope of Excel challenges and a second dataset to understand scalability issues specifically. 
The random dataset

Collection. To collect our first dataset, we used the Reddit API's "random" function, fetching 50-100 posts with each call and collected 278 posts in May of 2017 over the period of two weeks. With this data we aimed to understand the breadth of the issues users encountered.

Coding. We used open coding to code our random posts. Two authors individually examined 50-100 fetched posts at a time and developed criteria to separate the posts into themes (and then sub-themes). The two then compared their themes to create a codebook and a common set of themes. They repeated the process of fetching and categorizing posts until no new themes emerged.

Most of these posts related to a user's uncertainty of how to perform operations (e.g., how can one convert columns into rows) or described a behavior not meeting expectations (e.g., "AND" operation did not join two clauses in an array formula correctly, resulting in no data returned). Two posts described scalability issues, where the dataset size caused undesired behavior: in one, a formula calculated for $30 \mathrm{~min}$ utes before crashing, and in the second, Excel froze every time the screen shifted. We were intrigued by the scalability issues because (1) to date, they have not been chronicled in existing work, and (2) the outcomes of anecdotal cases did not have solutions. We, therefore collected a second dataset to explore scalability (see below).

Establishing Reliability. For both this and the following set, after creating the categories, two of the authors coded sets of $10 \%$ of the posts from each dataset separately until they achieved a Cohen's kappa of at least .78. They then proceeded to code the entirety of the dataset, comparing $5 \%$ of all post codes every 85 posts ( $20 \%$ of all posts), ensuring that they maintained a Cohen's kappa of at least .7.
The search term dataset

Collection. We used the Reddit API and searched for scalability posts via keywords within the same /r/excel forum. Adapting the methodology used in $[13,14,16]$, we finalized the set of search terms in the following manner: (1) From the randomly collected posts, we identified scalability posts and their keywords. (2) Using the keywords found in (1), we searched for additional scalability posts. We then extracted new scalability keywords from these posts. We repeated this process until no new keywords/phrases were identified. The final list of scalability keywords was "big", "crash", "forever", "freeze", "lag", "large", "long time", and "slow".

Using our final set of eight keywords/phrases, we created a dataset of 434 posts by collecting the first 100 posts returned by each keyword or until the API call ceased to return results, whichever happened first, from July through August of 2017 . Typically, approximately 70 posts were returned, but some keywords returned as few as nine.

While this approach was designed to filter scalability issues from the forum as a whole, we found that people also addressed Excel "crashing" or "freezing" in other situations (e.g., "freeze panes" or "crash course in Excel"). To capture posts that addressed the scalability issues, we created a codebook to separate scalability issues from non-scalability issues. We stopped collecting data when no new themes emerged in our coding of problem area themes.

Coding for Theme. We adapted the coding process we used for the random dataset to code this search term dataset. We ran each search term separately and coded resulting posts in batches as they arrived. Two of the authors developed criteria to separate the posts into themes (and then sub-themes). The two then compared their themes to create a codebook and a common set of themes. They repeated the process of fetching and categorizing posts un- 
til no new themes emerged. While we started with a blank slate after coding the random dataset, we found that the posts essentially fell into the same themes as the randomly fetched posts. The posts that did not fall into the four main themes were placed in a miscellaneous category.

Coding for Scalability. As described earlier, we separated the scalability related posts from the non-scalability related posts. The authors created the following criteria for this coding: a scalability issue is defined as poor behavior within an Excel workbook that contains a large amount of data. Specifically, poor behavior is characterized as Excel lagging, not responding, crashing, or taking long enough to respond that the user declared the spreadsheet unusable. The inclusion criteria for scalability posts were: (1) the user specified observing thousands of rows of data, (2) the user's file size was $5 \mathrm{MB}^{2}$ or larger, (3) the user did not quantitatively specify how large their data was, but they expressed suspicion that the issues were due to the dataset size, (4) a user could successfully import a small amount of data, but failed when the data size increased.

We coded the same dataset thematically and for scale. For both codings of the search term dataset, we established coding reliability in the same manner as we described in the random dataset coding section. From the 434 search term posts, 81 posts addressed scalability concerns, bringing the combined number of posts dealing with scalability issues to 83 out of 712 .

\section{The Themes}

We made two observations which laid out the architecture for our thematic coding. First, the posts were overwhelmingly questions, as opposed to statuses or updates. Sec-

\footnotetext{
${ }^{2}$ This is the size where users began experiencing "lag" and "freezing"
}

ond, the majority of the questions dealt with four themes: importing data, managing data, querying data, and presenting data. While the majority of posts fell into these four themes, the remainder were placed in a Miscellaneous theme. These posts generally asked broader, questions than other posts, as we discuss further below.

Our four main themes were further categorized into subthemes. We introduce the themes and sub-themes below with a brief description of the posts they contain, followed by a table presenting paraphrased quotes (to preserve anonymity) of posts from a selection of sub-themes. Each post received one of the following labels as well as a yes or no label indicating whether it related to scalability to better understand both the post issue whether the issue was affected by scalability. The pair of labels allowed us to see the operation areas affected by scalability. To illustrate how the "scalability" code changes the nature of the problems users encountered, we present several posts in Table 1 with their scalability code.

\section{Importing Data}

This theme includes posts related to importing data from sources such as CSV files, PDFs, websites, and other Excel workbooks.

\section{Managing Data}

This theme emerged from posts that discussed cleaning and updating data after it has been imported. This theme included the three sub-themes below.

Manipulating Data. This sub-theme includes questions about actions required to transform data into a analyzable state. (e.g., cleaning data after import and changing the format of data for a non-aesthetic purpose-changing the format of a date so it is consistent with existing data and formulas in the sheet.) 


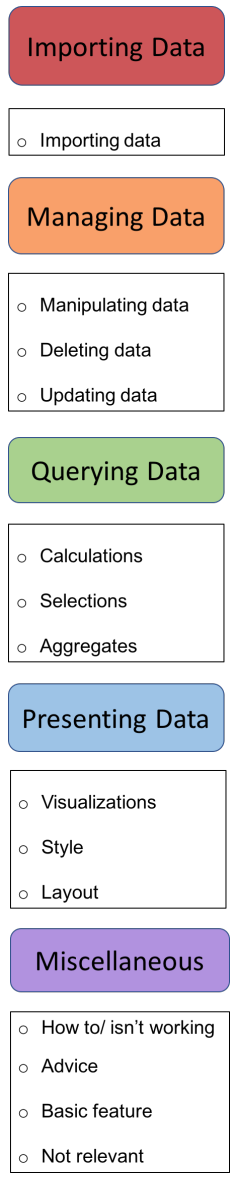

Figure 1: A hierarchical representation of the themes and their sub-themes.
Deleting Data. This sub-theme includes questions that ask how to delete or hide data from view.

Updating Data. This sub-theme includes questions asking how to update existing entries in a workbook or how to add more data to a workbook, e.g., adding a fixed value to all of the entries in a column or adding new entries to a list of records in a sheet.

\section{Querying Data}

This theme includes posts related to extracting information from the data in a workbook.

Calculations. This sub-theme includes questions on how to create new values from existing data in the spreadsheet. Note that simply selecting existing data from the sheet does not qualify as a calculation; new data must be created.

Selections. This sub-theme includes posts about finding and/or selecting pieces of information from a workbook (e.g., questions whose main topic is VLOOKUP). Other common examples discuss operations such as finding duplicates or finding which records fall between two dates.

Aggregations. This sub-theme includes posts that ask questions about grouping all records of a sheet into themes, counting entities (often via COUNTIF), summing entities, or using pivot tables. The main distinction between Aggregations and Selections is that Selections deal with selecting data that meets certain conditions while disregarding the remainder of the data, whereas Aggregations deal with organizing/categorizing all of the data in a sheet.

\section{Presenting Data}

This theme includes posts relating to how the data is presented to the Excel user (and not necessarily for the purpose of creating a formal presentation).
Visualizations. This sub-theme includes posts that relate to the creation of charts, graphs, and tables. The questions address creating templates, changing the style of a chart, or selecting the correct data to be used in a chart.

Style. This sub-theme includes posts that deal with the style of the Excel sheet or the style of the data within the sheet. Examples of style actions in the sheet include highlighting, outlining cells, and changing row/column widths. Actions that deal with the style of the data include changing font properties or the number of decimal places displayed.

Layout. This sub-theme includes posts that deal with the position or layout of data within the workbook. Questions asking how to move data within the same workbook also fall into this theme. Other common questions include moving all data in a certain manner (e.g., up one row or converting rows to columns).

\section{Miscellaneous}

These posts were significant enough to warrant coding, but did not fall into one of the aforementioned themes.

How-To or Isn't Working. This sub-theme included posts that either ask how to do a task (not present in the previous four themes) or ask why a simple feature in Excel does not meet user expectations. Often these formats of questions are combined into one-- $X$ is not working, how would I solve this problem". Creative uses of Excel (e.g., creating games within a spreadsheet) fell in this sub-theme.

Advice. This sub-theme includes posts that ask for general advice about Excel functionality. If a post was specifically asking about aggregating data, for example, we included it in the aggregate theme. However this theme captured questions that were broad in scope-for example, "how do I structure a large amount of data in a spreadsheet". 

Import
Manipulate
- Visualization
Style
Delete
Layout
Update
- Calculation
How to
- Selection
- Advice
Aggregation
Simple

Figure 2: The legend for the following charts.

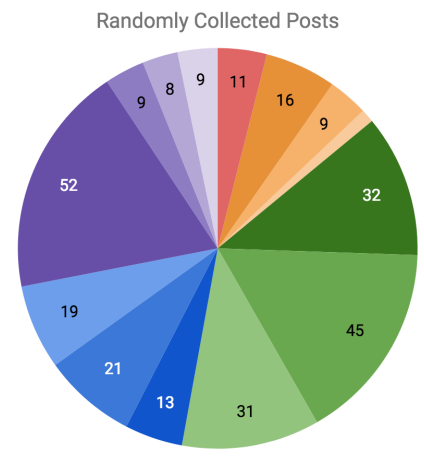

Figure 3: The distribution of the random posts (legend in figure Figure 2).
Simple Expected Features. This sub-theme includes posts that contain descriptions of simple features that did not function as expected. We define simple features as those required for Excel to properly function as data storage mechanism (e.g., opening/closing the application, adding a row to a sheet, saving the data, and scrolling).

\begin{tabular}{|l|l|l|}
\hline Category & Scalability & Quote \\
\hline Import & No & $\begin{array}{l}\text { "Is there a way I can automatically pull reports from } \\
\text { Google, Facebook, and email into an Excel file?" }\end{array}$ \\
\hline Import & Yes & $\begin{array}{l}\text { "I have over } 300 \text { Excel files. I would like to bring } \\
\text { them into one file, but it is tens of MB's large and it } \\
\text { does not conatin them all yet. Should I be using } \\
\text { something other than Excel?" }\end{array}$ \\
\hline Calculation & No & $\begin{array}{l}\text { "I want to add up how many hours I've worked } \\
\text { sporadically throught the day to get a day total." }\end{array}$ \\
\hline Calculation & Yes & $\begin{array}{l}\text { "In my 100,000 row spreadsheet, I have many } \\
\text { formulas to calculate ratios I need for a report. } \\
\text { However, now the file is large and extremely slow." }\end{array}$ \\
\hline Layout & No & "How do I freeze both columns and rows." \\
\hline Layout & Yes & $\begin{array}{l}\text { "I wrote some VBA code to help copy and post a } \\
\text { large dataset of 5K rows. I'm only trying it on a } \\
\text { couple hundred rows and it already crashes Excel." }\end{array}$ \\
\hline
\end{tabular}

Table 1: Excerpts from collected posts.

Not Relevant. This sub-theme includes posts that were not relevant to our case study addressing spreadsheet needs and desires. These posts included advertisements or requests for software alternatives to Excel.

\section{Results and Discussion}

The main goal of this work was to present the landscape of scalability issues present in Excel as captured in a popular subreddit forum. Figure 3 presents the distribution of the set of 278 random posts, Figure 4 presents the distribution of the 434 search term posts, and Figure 5 shows the distribution of the posts we categorized as relating to scalability. 83 of the 712 posts related to scalability. 81 came from the search terms posts, while the other two came from the random posts. We drew two main conclusions:

\section{Users understand the capabilities of Excel, but not} how to operationalize them. As a whole, the results stress the importance of Excel as a data storage tool and as a data processing engine (these posts account for $63.2 \%$ of all posts collected). Note the large difference between the number of posts that fell in the querying data $(29.5 \%$ of posts) theme compared to importing and manipulating data combined (13.3\% of posts). This indicates that users are generally comfortable with using Excel as a tool for storage, but they struggle when it comes to applying functions which allow them to extract useful information from their data.

In the majority of posts, users state what they would like Excel to do, and ask how to obtain this result. People do not underestimate what can be accomplished with Excel. The questions span the whole gamut of data analysis, from data collection (crawling the web with VBA code) to consumption of information (visualizations) and everything in between.

Many of the posts in the How to category deal with people trying to accomplish creative tasks with Excel. One user was trying to implement Tic-Tac-Toe.

These results together indicate that we need to make these complex and powerful capabilities more accessible to users. The technical knowledge of post makers ranged from people who were not familiar with VBA and wanted to avoid a solution using VBA to those who used it frequently.

Many of the posts tell a story of an often complex task the user wants to complete. Often these posts reflect an understanding of how to perform operations in Excel, but express confusion with stringing multiple operations together to perform a complete task. While documentation is very useful for understanding how a specific function works, it is not much help in understanding how to build a solution to a large problem out of basic functions. More research in the area of helping users understand how to integrate func- 


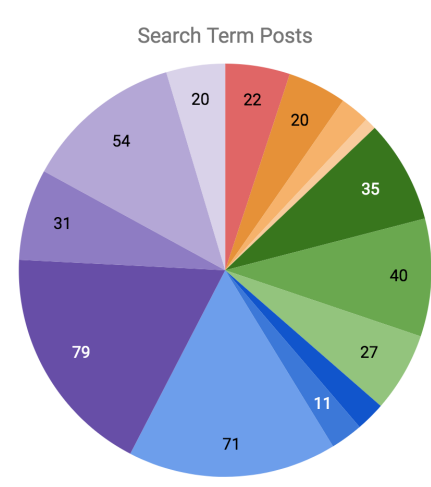

Figure 4: The distribution of the search term posts (legend in Figure 2).

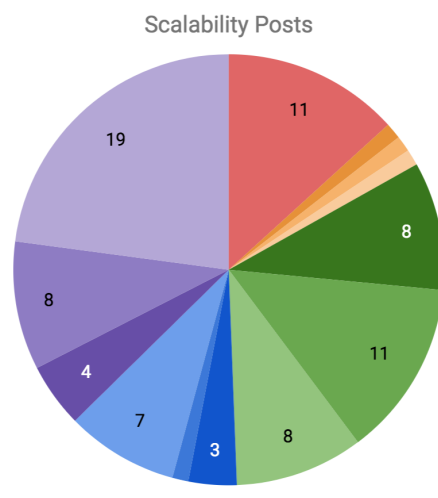

Figure 5: The distribution of the scalability posts (legend in Figure 2). tions together would be of great use to many of the post makers on the $/ r$ /excel subreddit. More information regarding suggested solutions to common questions can be found here [17].

\section{Scalability issues affect a wide range of operations} in spreadsheets. Every theme and sub-theme contains posts relating to scalability. Analyzing this distribution can help prioritize which operations are the most important to adapt to handle large quantities of data. For example, a large amount of the scalability issues deal with either importing data or querying data. These two areas in particular require further attention.

Notice that scalability issues cause a large number of the issues in the simple expected feature posts. This indicates that as data sets become larger, even the simplest of features in Excel cease to respond in a timely manner. Without these features, as the post makers comment, Excel is essentially useless. Users turn to other options like databases, but some are reluctant to use them because they are not familiar with SQL. Ideally, these users who are not familiar with databases should be able to use the spreadsheet interface they are comfortable with, while utilizing the processing power and scalability of a database.

Moreover, from an expressiveness point of view, many of the users' issues could be solved by a simple SQL statement. For example, one post maker asked "I want to be able to input two dates, and have shown the text and date for every date between those. I think i[sic] need to use some sort of array formula, but l'm not sure how (the exact formula, not array as a whole)". The suggested solution posted was to use the following formula: =IFERROR (INDEX ( $\mathrm{B}: \mathrm{B}, \mathrm{SMALL}(\mathrm{IF}((\$ B \$ 5: \$ B \$ 80>=\$ C \$ 34)$ * $(\$ 5: \$ B \$ 80<=\$ F \$ 28)$, $\operatorname{ROW}(\$ B \$ 5: \$ B \$ 80))$, ROWS(\$B\$5:\$B5))), "'") Not only is this formula hard to parse and understand, but also the post maker was not able to conceive of this formula themselves. They further could not alter the suggested formula for their task and needed assistance to organize the results. The solution to this problem is much simpler and cleaner in SQL: SELECT text, date FROM table WHERE date > date1 AND date < date2. Similar observations could be made of many of the questions in the querying data theme-that they could be expressible as simple SQL queries (with joins, group-by, and selections) $[1,5,19]$.

\section{Limitations}

Our methodology poses some limitations. Our collection approach using the "random" API call is described as a "the serendipity button," indicating that it returns a random post from the forum. However, verifying that it is truly random is challenging. In our results, we see a bias towards more recent posts. We also have a self-selection bias in the posters that contribute to the subreddit we studied.

\section{Conclusions}

In our case study, we set out to apply a method of utilizing online forum posts to understand usability issues people face with spreadsheets. We successfully identify four main categories of issues and multiple subthemes, leading us to believe the same method can be used to analyze other technologies to characterize usability issues. The majority of posts in the subreddit were Excel users asking questions about the four main steps needed to process data: importing, managing, querying, and presenting-we found that all four are important. Furthermore, not only are scalability issues very real problems plaguing those who want to analyze large amounts of data, but these issues touched on all four areas of actions users take to process data. This motivates the need for developing a spreadsheet application which continues to perform as expected on very large volumes of data [7]. 


\section{REFERENCES}

. Azza Abouzied, Joseph Hellerstein, and Avi Siberschatz. 2012. DataPlay: interactive tweaking and example-driven correction of graphical database queries. In Proceedings of the 25th annual ACM symposium on User interface software and technology. ACM, 207-218.

2. Michael Alexander, Jared Decker, and Bernard Wehbe. 2014. Microsoft business intelligence tools for Excel analysts. John Wiley \& Sons.

3. Lisa Anthony, YooJin Kim, and Leah Findlater. 2013. Analyzing user-generated youtube videos to understand touchscreen use by people with motor impairments. In Proceedings of the SIGCHI conference on human factors in computing systems. ACM, 1223-1232.

4. Salvatore Aurigemma and Ray Panko. 2014. Evaluating the Effectiveness of Static Analysis Programs Versus Manual Inspection in the Detection of Natural Spreadsheet Errors. Journal of Organizational and End User Computing

(JOEUC) 26, 1 (2014), 47-65. DOI:
http://dx.doi.org/10.4018/joeuc. 2014010103

5. Eirik Bakke, David Karger, and Rob Miller. 2011. A spreadsheet-based user interface for managing plural relationships in structured data. In Proceedings of the SIGCHI conference on human factors in computing systems. ACM, 2541-2550. DOT. http://dx.doi .org/10.1145/1978942.1979313

6. Eirik Bakke and David R Karger. 2016. Expressive query construction through direct manipulation of nested relational results. In Proceedings of the 2016 International Conference on Management of Data. ACM, 1377-1392.

7. Mangesh Bendre, Vipul Venkataraman, Xinyan Zhou, Kevin Chen-Chuan Chang, and Aditya Parameswaran 2017. Towards a Holistic Integration of Spreadsheets Ps: A Scalable Storage Engine for Pres (2017).

8. Chris Chambers and Chris Scaffidi. 2010. Struggling to excel: A field study of challenges faced by spreadsheet users. In Visual Languages and Human-Centric Computing (VL/HCC), 2010 IEEE Symposium on. IEEE, 187-194. DOI: http://dx.doi.org/10.1109/VLHCC.2010.33

9. David G Hendry and Thomas RG Green. 1994. Creating, comprehending and explaining spreadsheets: a cognitive interpretation of what discretionary users think of the spreadsheet model. International Joumal or Human-Computer Studies 40, 6 (1994), 1033-1065. DOI :http://dx.doi .org/https : //doi.org/10.1006/ijhc.1994.1047

10. Sun Hee Jang. 2011. YouTube as an innovative resource for social science ation for Research in Education Conference (AARE 2011 Conference). Citeseer, 1-16.

11. Jennifer Keelan, Vera Pavri-Garcia, George Tomlinson, and Kumanan Wilson. jama 298, 21 (2007), 2482-2484.

12. Swetha Keertipati, Bastin Tony Roy Savarimuthu, and Sherlock A. Licorish. 2016. Approaches for prioritizing feature improvements extracted from app reviews.. In EASE, Sarah Beecham, Barbara Klah ham, and Stephen G. $\mathrm{db} /$ conf/ease/ease2016. html\#KeertipatiSL16
13. Jennifer G Kim, Ha Kyung Kong, Karrie Karahalios, Wai-Tat Fu, and Hwajung Hong. 2016. The power of collective endorsements: credibility factors in medical Human Factors in Computing Systems. ACM, 4538-4549.

14. Danai Koutra, Paul N Bennett, and Eric Horvitz. 2015. Events and controversies: Influences of a shocking news event on information seeking In Proceedings of the 24th International Conference on World Wide Web. International World Wide Web Conferences Steering Committee, 614-624. DOI http://dx.doi.org/10.1145/2736277.2741099

15. Susan E Kruck and Steven D Sheetz. 2001. Spreadsheet accuracy theory. Journal of Information Systems Education 12, 2 (2001), 93-108. DOI:
http: //dx. doi .org/10.4018/joeuc . 2014010103

16. Juhi Kulshrestha, Motahhare Eslami, Johnnatan Messias, Muhammad Bila Zafar, Saptarshi Ghosh, Krishna P Gummadi, and Karrie Karahalios. 2017. Quantifying search bias: Investigating sources of bias for political searches in

social media. (2017), 417-432. DOI:
http://dx.doi.org/10.1145/2998181. 2998321

17. Kelly Mack, John Lee, Kevin Chang, Karrie Karahalios, and Aditya Parameswaran. 2017. Characterizing Scalability Issues in Spreadsheet Software using Online Forums. arXiv preprint arXiv:1801.03829 (2017). Technical report.

18. Roland Mittermeir and Markus Clermont. 2002. Finding high-level structures in spreadsheet programs. In Reverse Engineering, 2002. Proceedings. Ninth Working Conference on. IEEE, 221-232. DOI: http://dx.doi.org/10.1109/WCRE. 2002.1173080

19. Arnab Nandi and Michael Mandel. 2013. The interactive join: recognizing gestures for database queries. In CHl'13 Extended Abstracts on Human Factors in Computing Systems. ACM, 1203-1208.

20. Bonnie A Nardi and James R Miller. 1990a. An ethnographic study of distributed problem solving in spreadsheet development. In Proceedings of the 1990 ACM conference on Computer-supported cooperative work. ACM, 197-208. DOI : http://dx.doi.org/10.1145/99332.99355

21. Bonnie A Nardi and James R Miller. 1990b. The spreadsheet interface: A basis for end user programming. Hewlett-Packard Laboratories.

22. Ray Panko. 2016. What We Don't Know About Spreadsheet Errors Today: The Facts, Why We Don't Believe Them, and What We Need to Do. arXiv preprint arXiv:1602.02601 (2016).

23. Stephen G Powell, Kenneth R Baker, and Barry Lawson. 2008. A critical review of the literature on spreadsheet errors. Decision Support Systems 46, 1 (2008), 128-138. DOI:

http://dx.doi.org/10.1016/j.dss.2008.06.001

24. Kamalasen Rajalingham, David R Chadwick, and Brian Knight. 2008. Classification of spreadsheet errors. arXiv preprint arXiv:0805.4224 (2008).

25. Thomas Reschenhofer and Florian Matthes. 2015. An empirical study on spreadsheet shortcomings from an information systems perspective. In International Conference on Business Information Systems. Springer, 50-61. 